\title{
Using anisotropies in prompt fission neutron coincidences to assess the neutron multiplication of highly multiplying subcritical plutonium assemblies
}

\author{
J. M. Mueller*, J. Mattingly \\ Department of Nuclear Engineering, North Carolina State University, Raleigh, NC 27695, \\ $U S A$
}

\begin{abstract}
There is a significant and well-known anisotropy between the prompt neutrons emitted from a single fission event; these neutrons are most likely to be observed at angles near $0^{\circ}$ or $180^{\circ}$ relative to each other. However, the propagation of this anisotropy through different generations of a fission chain reaction has not been previously studied. We have measured this anisotropy in neutron-neutron coincidences from a subcritical highly-multiplying assembly of plutonium metal. The assembly was a $4.5 \mathrm{~kg} \alpha$-phase plutonium metal sphere composed of $94 \%$ ${ }^{239} \mathrm{Pu}$ and $6 \%{ }^{240} \mathrm{Pu}$ by mass. Data were collected using two EJ-309 liquid scintillators and two EJ-299 plastic scintillators. The angular distribution of neutron-neutron coincidences was measured at $90^{\circ}$ and $180^{\circ}$ and found to be largely isotropic. Simulations were performed using MCNPX-PoliMi of similar plutonium metal spheres of varying sizes and a correlation between the neutron multiplication of the assembly and the anisotropy of neutron-neutron coincidences was observed. In principle, this correlation could be used to assess the neutron multiplication of an unknown assembly.
\end{abstract}

Keywords: Passive interrogation; Plutonium; Nonproliferation; Special nuclear material; Neutron angular anisotropy; Neutron detectors

\footnotetext{
${ }^{*}$ Corresponding author

Email address: jonathan_mueller@ncsu.edu (J. M. Mueller)
}

Preprint submitted to Nuclear Instruments and Methods in Physics Research Section AApril 4, 2016

(c) 2016. This manuscript version is made available under the Elsevier user license http://www.elsevier.com/open-access/userlicense/1.0/ 


\section{Introduction}

Characterizing the neutron multiplication of unknown assemblies of special nuclear material (SNM) is an important area of research for nuclear arms dismantlement verification. The multiplication of an assembly is defined as the total expected number of neutrons created within the assembly for one incident or source neutron. Sources that do not sustain fission chain reactions, such as AmBe or ${ }^{252} \mathrm{Cf}$, can be distinguished from sources capable of sustaining fission chain reactions, such as weapons-grade plutonium or highly-enriched uranium, by measurements of the multiplication of the source.

A widely-used technique for measuring the multiplication of an assembly of SNM is neutron multiplicity counting. In this technique, ${ }^{3} \mathrm{He}$ proportional counters surrounded by a neutron moderator such as high density polyethylene are used to measure neutron singles, double coincidences, and triple coincidences. The multiplication of the assembly can be inferred based on these count rates and fundamental nuclear data parameters [1]. Despite the success of this technique, there are several disadvantages to this method. One significant disadvantage is that the neutrons from the source must be thermalized to be detected with acceptable efficiency in the ${ }^{3} \mathrm{He}$ proportional counters. The thermalization process obscures some information about the neutrons emitted by the source, such as their original energies and emission directions.

Here, we propose to use the anisotropy of coincident prompt fission neutrons to assess the multiplication of a subcritical assembly of plutonium. Experimental measurements and simulations will show that the anisotropy of coincident prompt fission neutrons depends on the multiplication of the subcritical assembly. Specifically, it will be demonstrated that the prompt neutron coincidences are anisotropic for bare spheres of weapons-grade plutonium with neutron multiplications below 3, and the coincidences are isotropic for these materials with multiplications above 3 .

In nuclear arms dismantlement verification, it is critical to prevent the disso closure of sensitive details about the nuclear material itself, such as its mass, 
multiplication, or geometry. Other techniques have focused on using information barriers or zero-knowledge proofs [2] to prevent the disclosure of this classified information. However, in this case the information barrier is related to the fundamental physics of the process itself, since any neutron multiplication above a critical value will result in an isotropic coincidence angular distribution. Therefore, the actual value of the multiplication cannot be recovered from measuring the coincidence angular distribution; all that will be known to inspectors is that the material had a large neutron multiplication. This physics-based information barrier could be particularly useful for nuclear arms dismantlement verification.

Section 2 describes some of the measurements and theory of the neutronneutron coincidence anisotropy and projects how this anisotropy might propagate in fission chain reactions. Section 3 discusses an experimental measurement of a highly multiplying subcritical assembly of plutonium. The analysis and results of that experiment, and MCNPX-PoliMi and GEANT4 simulations of the experimental setup, are provided in Section 4. Results from simulations of spherical plutonium assemblies of varying sizes are discussed in Section 5 Finally, conclusions based on the present studies are given in Section 6 .

\section{Previous work on prompt fission neutron coincidence anisotropy}

The angular distribution of coincident prompt fission neutrons was first measured in Ref. [3]. In that experiment, fast neutrons induced fission within a sample of ${ }^{235} \mathrm{U}$, and the prompt neutron coincidence count rate was measured for two detectors as a function of the relative angle between the detectors. They observed a higher coincidence rate when the detectors were placed at $180^{\circ}$ relative to each other than when they were placed at $90^{\circ}$. Since that initial experiment, many additional measurements of the angular dependence of prompt neutron coincidences have been performed for spontaneous fission of ${ }^{252} \mathrm{Cf}[4,[5,6]$ and neutron induced fission of ${ }^{235} \mathrm{U}$ [7]. The general observation of these measurements is that prompt fission neutrons from the same fission event are correlated in angle. They are most likely to be observed at $0^{\circ}$ or $180^{\circ}$ relative to each other 
and least likely to be observed at $90^{\circ}$ relative to each other. This anisotropy in the neutron-neutron coincidences has a demonstrated applicability in extracting the ratio of the spontaneous fission rate to the $(\alpha, \mathrm{n})$ rate in oxide samples of SNM 8 .

The cause of the angular correlation between the neutrons is evident when examining the time scale for the predominant method of generating prompt neutrons. The majority of the prompt neutrons are emitted after the fragments have nearly fully accelerated 9 . It is generally assumed that the neutrons are emitted with no preferred direction in the rest frame of each fragment. However, because the fragments have already nearly fully accelerated, the prompt neutrons tend to travel in the direction of the fission fragments. The fission fragments themselves travel at $180^{\circ}$ with respect to each other because they are accelerated due to their mutual Coulomb repulsion. Therefore, the neutrons emitted from the same fragment will tend to appear at angles near $0^{\circ}$ relative to each other, and the neutrons emitted from complementary fragments will tend to appear at angles near $180^{\circ}$ relative to each other.

The angular dependence of the prompt neutron coincidences also depends on the prompt neutron energies. Neutrons traveling in the same direction as the fission fragment will receive a larger velocity boost into the lab frame, gaining more energy. Therefore, the higher energy neutrons will have a stronger angular correlation than the lower energy neutrons. This effect has been observed in Refs. 4, 5, and has been accurately reproduced in an advanced Monte Carlo simulation [10].

While this angular correlation and its energy dependence is a clear signature of a single fission event, the propagation of this anisotropy in a fission chain reaction has not been previously studied. Fundamentally, the propagation of this anisotropy in a fission chain reaction depends significantly on the fission fragment angular distribution for neutron-induced fission. The fission fragment angular distribution has been measured for fast-neutron-induced fission of ${ }^{235} \mathrm{U}$ [11, 12, 13] and fast-neutron-induced fission of ${ }^{239} \mathrm{Pu}$ [13]. Those measurements 90 showed that it was slightly more likely, approximately $10-20 \%$ depending on the 
isotope and neutron energy, for the fission fragments to be emitted in the direction of the incident neutron rather than perpendicular to the direction of the incident neutron. This small angular correlation between the incident neutron and fission fragments becomes even weaker when considering the angular correlation between the incident neutron and the prompt neutrons generated from these fission fragments. Based on these angular distributions, it is expected that neutrons from different generations will be largely uncorrelated in angle.

For assemblies of SNM with low multiplication, most of the coincident neutrons will be from single fission events, so they will have a strong anisotropy. However, for assemblies of SNM with high multiplication, a higher proportion of the coincident neutrons will be from different generations of a fission chain reaction, so the anisotropy should decrease significantly. Based on the preceding physical arguments, we have investigated the possibility of using the prompt neutron coincidence anisotropy to assess the multiplication of an assembly of SNM.

\section{Description of the experiment}

Prompt neutron coincidence anisotropy measurements were performed using a plutonium assembly located at the Device Assembly Facility (DAF) on the Nevada National Security Site (NNSS). The plutonium assembly, hereafter referred to as the BeRP ball, is a $4.5 \mathrm{~kg}$ sphere of $\alpha$-phase plutonium metal. The radius of the BeRP ball is approximately $3.8 \mathrm{~cm}$ and it is encased in stainless steel with a nominal thickness of $0.3 \mathrm{~mm}$. It is composed of approximately $93.3 \%{ }^{239} \mathrm{Pu}, 5.9 \%{ }^{240} \mathrm{Pu}$, and $0.2 \%{ }^{241} \mathrm{Am}$ by mass [14. The primary neutron source is the spontaneous fission of ${ }^{240} \mathrm{Pu}$ within the assembly itself, and this generates approximately $2.8 \times 10^{5}$ neutrons per second which can then induce fission chain reactions. The $(\alpha, \mathrm{n})$ rate from the $\mathrm{BeRP}$ ball is negligible. The multiplication of the BeRP ball is 4.5 .

The setup of the experiment is shown in Figure 1 . The BeRP ball is located at the center of the figure on top of a small aluminum stand. The data presented 


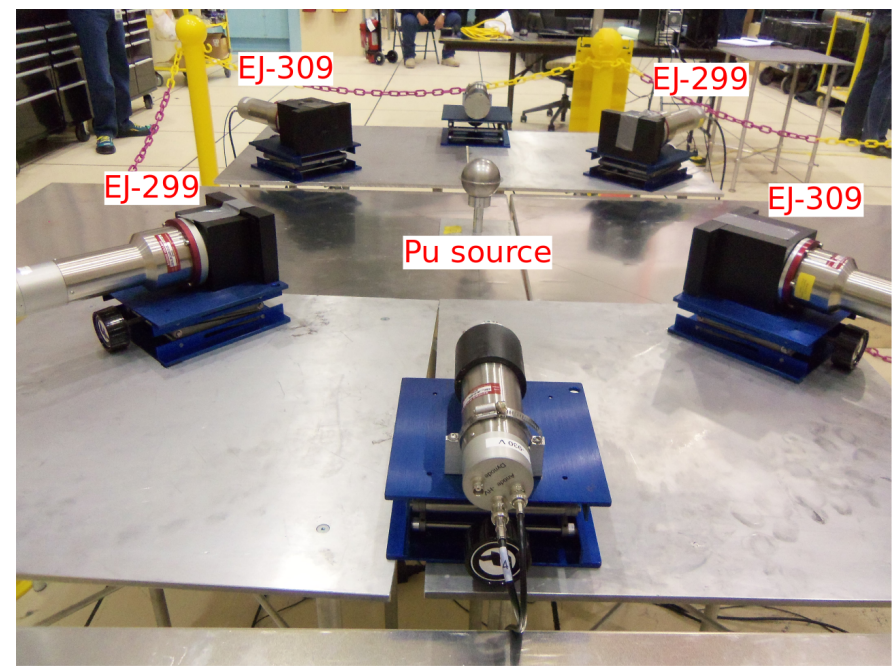

Figure 1: The experimental setup used to measure neutrons from the BeRP ball.

120

two EJ-299 plastic organic scintillators. All four of these detectors had active volumes that were $7.6 \mathrm{~cm}$ in diameter and $7.6 \mathrm{~cm}$ long. The four detectors were placed approximately $52.5 \mathrm{~cm}$ from the source. The two EJ-309 scintillators were placed on opposite sides of the source, and the two EJ-299 scintillators were also placed on opposite sides of the source and perpendicular to the line between the centers of the EJ-309 detectors.

The BeRP ball is a strong gamma-ray emitter due primarily to the high content of ${ }^{241} \mathrm{Am}$. Without any shielding, these gamma rays could overwhelm the PSD capabilities of the scintillators. Therefore, all detectors were shielded to reduce the number of gamma-ray events. All four detectors had $3.2 \mathrm{~cm}$ of lead shielding in front of the active volumes and $0.6 \mathrm{~cm}$ of lead shielding around their circumference to shield them from scattered gamma rays.

Calibration measurements were performed with a ${ }^{252} \mathrm{Cf}$ source and a ${ }^{137} \mathrm{Cs}$ source. For both sources, measurements were taken both with $0.6 \mathrm{~cm}$ and 3.2 $\mathrm{cm}$ of lead shielding in front of the detectors. The measurements using the ${ }^{137} \mathrm{Cs}$ source were performed to calibrate the detector responses using the calibration procedure outlined in Refs. [15, 16. The coincident prompt gamma rays from 


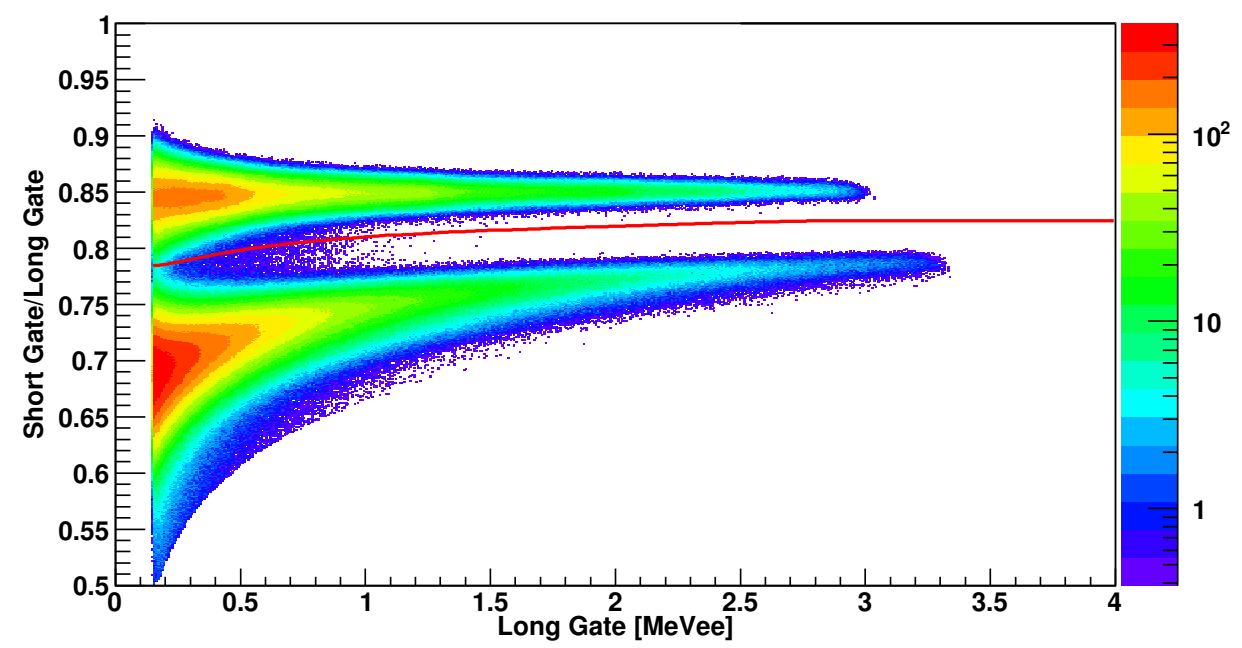

Figure 2: The ratio of short-gate to long-gate integrals is shown as a function of the long-gate integral for one of the EJ-309 detectors. The red line discriminates between the gamma-ray band (top) and the neutron band (bottom).

the ${ }^{252} \mathrm{Cf}$ source were used to calibrate the relative timings between the detectors to account for small differences in photomultiplier tube (PMT) transit times, detector distances, and cable lengths. Coincident prompt neutrons from the ${ }^{252} \mathrm{Cf}$ source are expected to exhibit a significant anisotropy, so the ${ }^{252} \mathrm{Cf}$ source was also used to ensure that the measurement system could accurately detect the presence of neutron coincidence anisotropy.

Full waveforms from the detectors were recorded using a CAEN DT5730B digitizer, which has 14-bit resolution and a $500 \mathrm{MHz}$ sample rate. The data acquisition software is described in Ref. [17]. A fiber optic connection was used to maximize the throughput of the digitizer and record events up to $80 \mathrm{MB} / \mathrm{s}$ with no dead time. The waveforms were processed offline to extract three quantities for each well-separated pulse in each waveform: a short-gate integral, long-gate integral, and trigger time using a digital constant fraction discrimination (CFD) algorithm. Pulses that appeared near the end of a digitized waveform or overlapped with another pulse were rejected. Pulse shape discrimination (PSD) 
based on the short-gate and long-gate integrals was used to distinguish neutron events from gamma-ray events. A representative PSD histogram for one of the EJ-309 detectors is shown in Figure 2. The typical PSD figure of merit, given by the difference between the centroid of the neutron and gamma-ray bands divided by the sum of their full widths at half maxima [18], is 1.33 for the EJ-309 detectors and 0.920 for the EJ-299 detectors, at thresholds of 150 and 140 keVee respectively.

\section{Experimental analysis and results}

After analyzing the waveforms, the prompt neutron coincidence anisotropy was determined. Let subscripts 1 and 3 indicate the two EJ-309 detectors and let subscripts 2 and 4 indicate the two EJ-299 detectors. The relative number of coincident neutrons at $180^{\circ}$ is given by:

$$
W\left(180^{\circ}\right)=\frac{1}{2}\left(\frac{D_{13}}{S_{1} S_{3}}+\frac{D_{24}}{S_{2} S_{4}}\right),
$$

where $D_{i j}$ is the number of prompt neutron coincidences in detectors $i$ and $j$, and $S_{i}$ is number of neutron singles in detector $i$. The coincidence rate is scaled in Equation 1 by the product of the singles rates to cancel out the effects of differences in detection efficiencies and solid angles. Similarly, the relative number of coincident neutrons at $90^{\circ}$ is given by:

$$
W\left(90^{\circ}\right)=\frac{1}{4}\left(\frac{D_{12}}{S_{1} S_{2}}+\frac{D_{14}}{S_{1} S_{4}}+\frac{D_{23}}{S_{2} S_{3}}+\frac{D_{34}}{S_{3} S_{4}}\right) .
$$

As only four detectors were used in a fixed configuration, it was not possible to measure a full angular distribution. However, the anisotropy in the angular distribution was assessed by measuring the angular asymmetry between $90^{\circ}$ and $180^{\circ}$, given as:

$$
\text { Asym }=\frac{W\left(180^{\circ}\right)-W\left(90^{\circ}\right)}{W\left(180^{\circ}\right)+W\left(90^{\circ}\right)}
$$

The angular asymmetry is bounded by -1 and 1 , and an asymmetry of 0 equates to an equal probability of coincidences at $90^{\circ}$ and $180^{\circ}$. 
A coincidence window of $200 \mathrm{~ns}$ was used for all of the measurements. The choice of coincidence window depends on several factors, including the detector distance and source activity. A coincidence window that is too short will reduce the statistical precision of the results and slightly bias the coincidences towards larger neutron energies, which will cause the asymmetry to increase slightly. A coincidence window that is very long will include a significant proportion of accidental coincidences, which will decrease the asymmetry. For this source activity and placement of detectors, we found that 200 ns was a reasonable coincidence window width, though the asymmetry was largely insensitive to a wide range of coincidence window widths.

Based on the discussion in Section 2, for low levels of multiplication the angular asymmetry should be significantly greater than 0 . However, as the multiplication of the assembly increases, the asymmetry should decrease to 0 . We do not expect to observe negative values of the asymmetry for symmetric geometries. Finally, the asymmetry should increase with increasing neutron energy.

Figure 3 shows the experimental measurement and Monte Carlo simulations of the angular asymmetry from the bare ${ }^{252} \mathrm{Cf}$ source as a function of detector threshold. Note that the threshold here is expressed in $\mathrm{MeV}$ and not in the traditional $\mathrm{MeV}$ electron equivalent (MeVee). The use of $\mathrm{MeV}$ instead of MeVee was motivated by the fact that two different scintillators were used in the experiment: EJ-309 and EJ-299. The light output from these scintillators is different for the same neutron energy, so adjusting the threshold in MeVee uniformly across all four detectors would have been an inaccurate representation of the same neutron energies. Instead, the threshold was adjusted by choosing a particular neutron energy in $\mathrm{MeV}$ and calculating the maximum light output in both scintillators using the measured detector responses from Ref. [15]. This procedure was motivated by the discussion of neutron energy-equivalent thresholds in Ref. [15].

Simulations of our experimental setup were performed in MCNPX-PoliMi [19] and GEANT4 20]. For the simulations using MCNPX-PoliMi, the ENDF/B- 


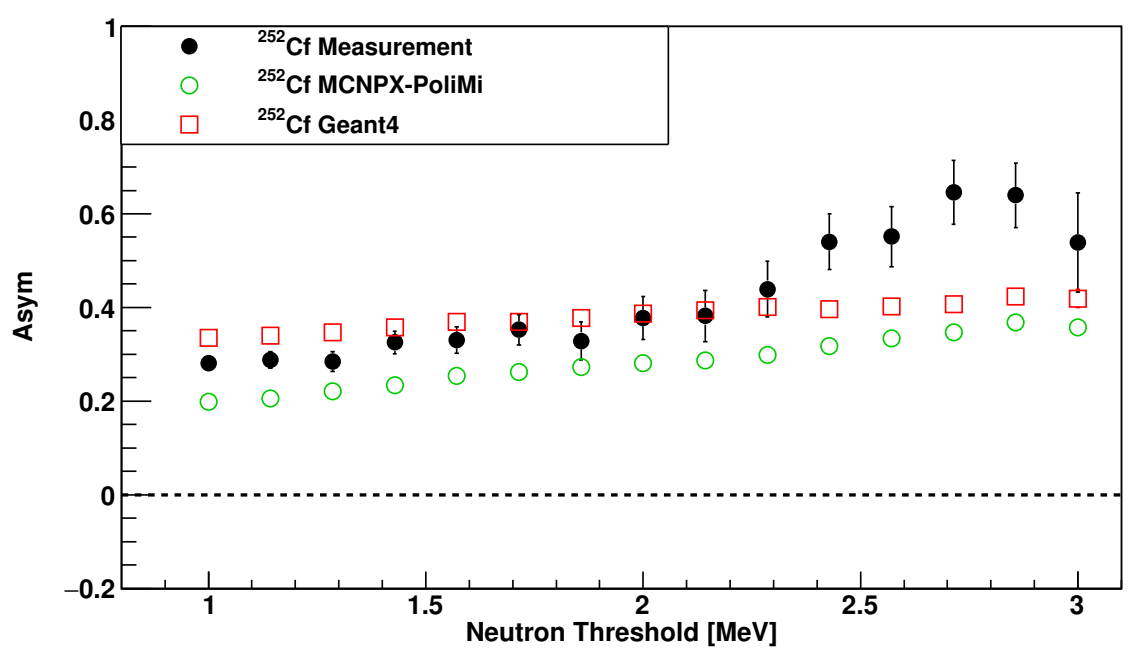

Figure 3: The angular asymmetry from the ${ }^{252} \mathrm{Cf}$ source is shown as a function of detector threshold. Uncertainties shown are statistical only, but are correlated between different data points of the same data set. Uncertainties that are not visible are smaller than the size of the data points.

VII.1 data libraries were used. The geometry in the MCNPX-PoliMi simulation included either the ${ }^{252} \mathrm{Cf}$ source or the BeRP ball, the source stand, the surface of the table, the floor, the lead shielding around the detectors, and the active region of the scintillating volumes.

The GEANT4 simulations were performed using the ENDF/B-VII.0 data library as enabled by Ref. 21] and the FREYA fission primary generator as described in Ref. [22]. The GEANT4 simulation used an identical geometry to the MCNPX-PoliMi simulations except the floor was omitted. Ref. [17] contains more information regarding the MCNPX-PoliMi and GEANT4 simulations.

The results shown in Figure 3 demonstrate that the spontaneous fission of ${ }^{252} \mathrm{Cf}$ shows a significant angular asymmetry and that our measurement system is capable of detecting this asymmetry. The asymmetry increases as the detector threshold increases, which agrees with our expectations based on Section 2. The MCNPX-PoliMi simulation predicts a smaller asymmetry than observed, while 


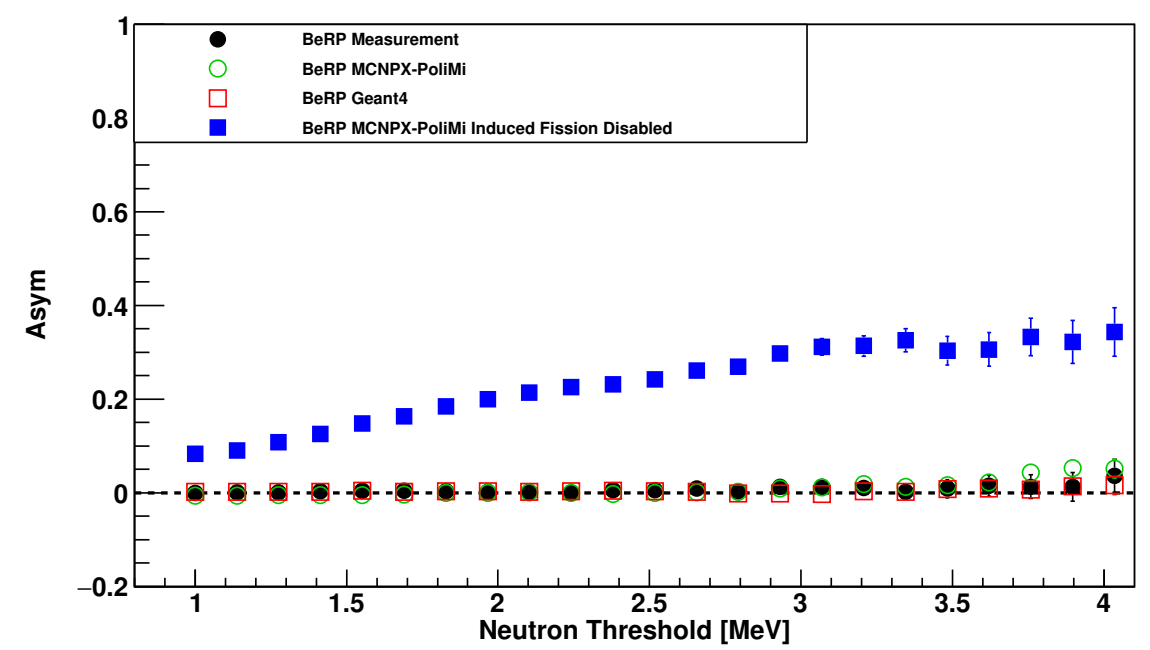

Figure 4: The angular asymmetry from the BeRP Ball is shown as a function of detector threshold. Uncertainties shown are statistical only, but are correlated between different data points of the same data set. Uncertainties that are not visible are smaller than the size of the data points.

the GEANT4 simulation predicts a slightly larger asymmetry than observed at low neutron thresholds.

Figure 4 shows the experimental measurement and Monte Carlo simulations of the angular asymmetry from the BeRP ball as a function of detector threshold. The experimental measurement of the BeRP ball shows almost no angular asymmetry over all of the different neutron thresholds. At a threshold of $4 \mathrm{MeV}$, the asymmetry may have a small positive value, but otherwise it is largely consistent with zero. This result is consistent with our expectations for a highly multiplying assembly. Overall, both the GEANT4 simulation and MCNPX-PoliMi simulation agree well with our measured angular asymmetry. The good agreement, combined with the fact that the simulations were able to model the asymmetry for the ${ }^{252} \mathrm{Cf}$ source, suggests that these simulations are able to model the effect of multiplication on the prompt neutron coincidence anisotropy. 
Two simulations of the experimental setup were performed in MCNPXPoliMi: one with induced fission enabled and an identical simulation except with induced fission disabled. The simulation in MCNPX-PoliMi with induced fission disabled should reasonably demonstrate the sole impact of induced fission on the prompt neutron angular asymmetry. This simulated asymmetry is significantly larger than 0 and increases with increasing neutron energy threshold, as expected for fission neutrons. Furthermore, this asymmetry is sizable; for a neutron energy threshold of $2 \mathrm{MeV}$, approximately $50 \%$ more coincident neutrons were detected in the simulation at $180^{\circ}$ than at $90^{\circ}$. This simulation demonstrates that effects other than multiplication, such as the finite size of the assembly and detectors, the multiple scattering of neutrons within the assembly, or accidental coincidences between neutrons from different fission chain reactions, do not account for the resulting measured angular asymmetry. The multiplication of the BeRP ball plays a significant role in the angular asymmetry.

\section{Simulations of similar assemblies}

The experimental results demonstrate a significant angular asymmetry for the ${ }^{252} \mathrm{Cf}$ source, which has a multiplication of 1.0, and no significant angular asymmetry for the BeRP ball, which has a multiplication of 4.5. Therefore, there must be some range of multiplications between 1.0 and 4.5 in which the angular asymmetry decreases from a non-zero value to zero.

Several MCNPX-PoliMi simulations were performed to investigate the range of multiplication between 1.0 and 4.5. These simulations were identical to the MCNPX-PoliMi simulation of the BeRP ball described in Section 4, except the radius of the plutonium sphere was varied between $0.01 \mathrm{~cm}$ and $3.75 \mathrm{~cm}$. The size of the assembly was used as a proxy to vary the multiplication. As the radius of the sphere decreased, the probability for neutrons to escape the sphere increased, thereby decreasing the multiplication.

Figure 5 shows the simulated asymmetry as a function of the radius of the 


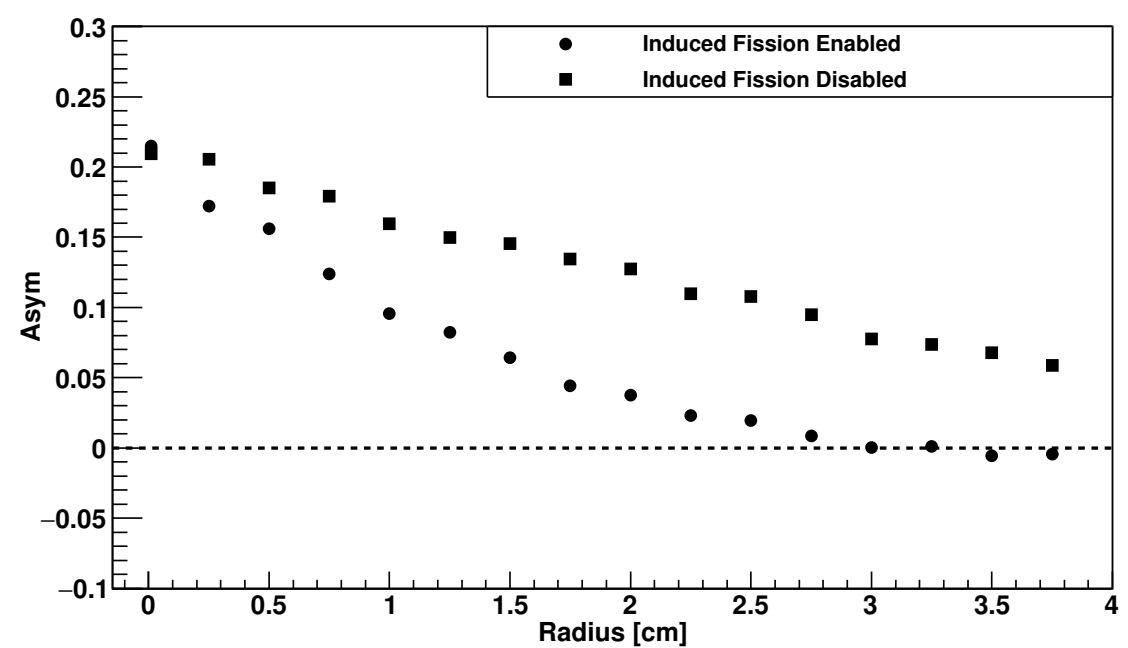

Figure 5: The angular asymmetry is shown as a function of the radius of the simulated plutonium assembly. Uncertainties are statistical only and are smaller than or approximately the same size as the data points.

assembly. The neutron threshold in the simulation is $1.0 \mathrm{MeV}$. The angular asymmetry for point-like assemblies is large, and the asymmetry smoothly decreases as the radius of the assembly increases.

However, neutron multiple scattering will impact the angular asymmetry and change with assembly size. As the size of the assembly increases, the probability of neutron multiple scattering increases, which would decrease the asymmetry without any induced fission. Therefore, two simulations were performed for each radius: one with induced fission enabled and another with induced fission disabled. The comparison between the two simulations shows the impact of the neutron multiplication effect alone. On average, over all of the assembly sizes, the neutron multiplication accounts for approximately half of the decrease in angular asymmetry compared to a point-like source, while other effects such as neutron multiple scattering account for the other half.

Figure 6 shows the simulated asymmetry as a function of the multiplication for different neutron energy thresholds. There is a clear trend of decreasing 


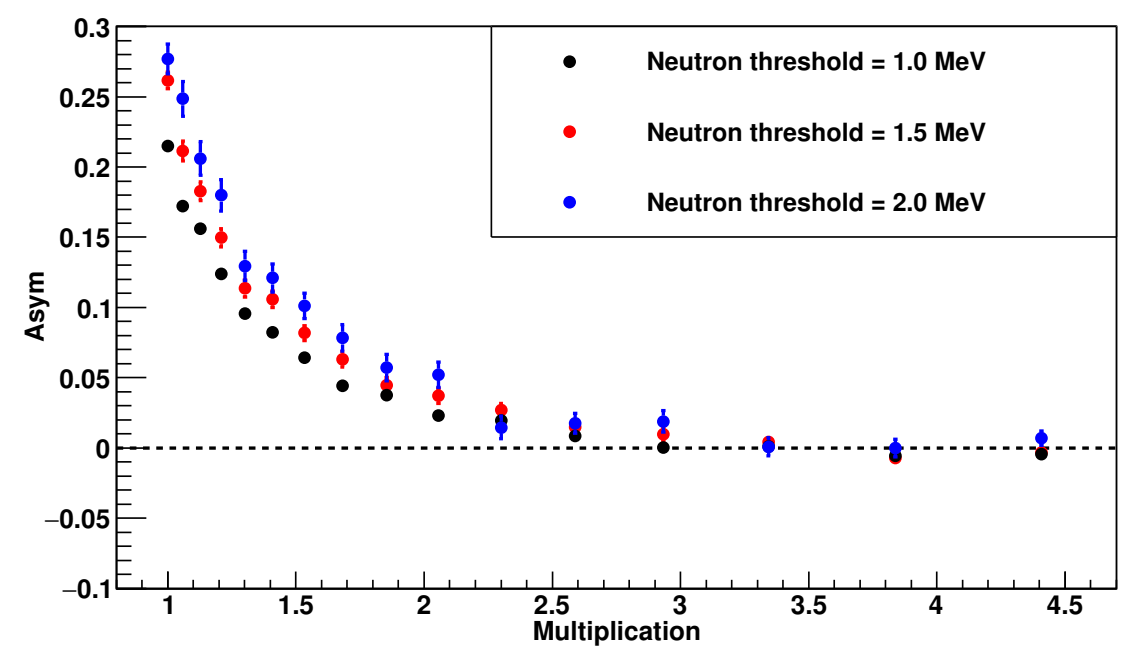

Figure 6: The angular asymmetry is shown as a function of the multiplication of the simulated plutonium assembly. Uncertainties are statistical only and when not shown are smaller than the data points.

asymmetry with increasing multiplication. For a neutron threshold of $1.0 \mathrm{MeV}$, the asymmetry is consistent with 0 for multiplications above 3 . The asymmetry can be increased by raising the neutron energy threshold, as is shown from the calculations with thresholds of $1.5 \mathrm{MeV}$ and $2.0 \mathrm{MeV}$. However, this increased sensitivity reduces the statistical precision of the simulated results.

\section{Conclusions and future work}

A novel study of the dependence of the prompt neutron coincidence angular distribution on the multiplication of an assembly of SNM was performed. For non-multiplying sources, previous work has established a significant anisotropy in prompt fission neutron coincidences. However, this angular correlation is expected to propagate only weakly, if at all, through a fission chain reaction. Therefore, neutrons from different fission events in the same chain reaction should be largely uncorrelated in angle. An experimental measurement of the anisotropy in prompt neutron coincidences was performed using a highly multi- 
plying source for the first time. The anisotropy was characterized by measuring the angular asymmetry between coincidences at $90^{\circ}$ and $180^{\circ}$. No significant asymmetry was measured for this source, in agreement with simulations performed using MCNPX-PoliMi and GEANT4.

Additional simulations were performed where the size of the plutonium assembly was varied; these simulations revealed the dependence of the angular asymmetry on the multiplication of the assembly. As the multiplication of the simulated assembly increased, a higher proportion of coincidences were between different generations of fission chain reactions, reducing the angular asymmetry. The results of these simulations suggest that an assessment of the multiplication could be made based on a measurement of the angular asymmetry. For multiplications of the weapons-grade plutonium assembly between 1 and 3 , the multiplication appears to be uniquely correlated to an asymmetry. For multiplications above 3 , the asymmetry appears to vanish.

This effect could be useful in cases where one needs to verify that an unknown assembly has a multiplication greater than 3 , but the precise value of the multiplication should not be revealed, which is similar to constraints placed on measurements for weapons dismantlement verification. In weapons dismantlement verification, it is imperative that classified information not be revealed to the inspectors. In this case, the coincidence asymmetry acts as a physicsbased information barrier that prevents the inspectors from obtaining sensitive information, while allowing the inspectors to ensure that the assembly is indeed composed of highly multiplying SNM.

There are several possibilities to expand on the present work. One critical step is to experimentally verify the dependence of the prompt neutron coincidence anisotropy on the multiplication, as currently this dependence is given only by MCNPX-PoliMi simulations. Another important consideration 320 is how the presence of shielding, such as polyethylene or lead, might affect the anisotropy and distort the relationship between the anisotropy and the multiplication. In addition to examining the impact of neutron reflectors, the dependence of the coincidence anisotropy on the plutonium grade or uranium 
enrichment should be investigated. It is possible that the addition of neutron reflectors or changing the plutonium grade or uranium enrichment will change the multiplication at which the neutron coincidences become isotropic. Finally, future work should assess the anisotropy from sources which are not cylindrically symmetric, since an asymmetric geometry may impact the anisotropy.

\section{Acknowledgements}

The authors would like to thank the staff at the NNSS for helping us perform this experiment at the DAF. The authors would also like to thank the staff provided by Los Alamos National Laboratory, and in particular Jesson Hutchinson, for helping us schedule and coordinate this experiment at the DAF. This work is sponsored by the NNSA Office of Defense Nuclear Nonproliferation R\&D through the Consortium for Nonproliferation Enabling Capabilities (CNEC) and the Consortium for Verification Technology (CVT).

\section{References}

[1] D. Cifarelli, W. Hage, Nuclear Instruments and Methods in Physics Research Section A: Accelerators, Spectrometers, Detectors and Associated Equipment 251 (1986) 550-563. doi:10.1016/0168-9002(86)90651-0.

口 [2] A. Glaser, B. Barak, R. J. Goldston, Nature 510 (2014) 497-502. doi 10. 1038/nature13457.

[3] S. Debenedetti, J. E. Francis, W. M. Preston, T. W. Bonner, Physical Review 74 (1948) 1645-1650. doi 10.1103/PhysRev.74.1645.

[4] S. Pozzi, et al., Nuclear Science and Engineering 178 (2014) 250-260. doi:10.13182/NSE13-96

[5] A. M. Gagarski, et al., Bulletin of the Russian Academy of Sciences: Physics 72 (2008) 773-777. doi:10.3103/S1062873808060130. 
[6] J. S. Pringle, F. D. Brooks, Physical Review Letters 35 (1975) 1563-1566. doi:10.1103/PhysRevLett.35.1563

[7] C. Franklyn, C. Hofmeyer, D. Mingay, Physics Letters B 78 (1978) 564-567. doi $10.1016 / 0370-2693(78) 90640-8$.

[8] L. Holewa, W. Charlton, E. Miller, S. Pozzi, Nuclear Instruments and Methods in Physics Research Section A: Accelerators, Spectrometers, Detectors and Associated Equipment 701 (2013) 249-253. doi:10.1016/j. nima.2012.11.026.

[9] A. Vorobyev, et al., Nuclear Instruments and Methods in Physics Research Section A: Accelerators, Spectrometers, Detectors and Associated Equipment 598 (2009) 795-801. doi:10.1016/j.nima.2008.10.017.

360 [10] R. Vogt, J. Randrup, Physical Review C 90 (2014) 064623. doi 10.1103/ PhysRevC.90.064623

[11] J. W. Meadows, C. Budtz-Jørgensen, in: Nuclear Data for Science and Technology: Proceedings of the International Conference, Antwerp, 1982, p. 740.

[12] S. Ahmad, et al., Nuclear Science and Engineering 71 (1979) 208.

[13] J. E. Simmons, R. L. Henkel, Physical Review 120 (1960) 198-210. doi:10. 1103/PhysRev.120.198.

[14] J. Mattingly, Polyethylene-Reflected Plutonium Metal Sphere: Subcritical Neutron and Gamma Measurements, Sandia National Laboratories SAND2009-5804-R3, 2009.

[15] C. C. Lawrence, et al., Nuclear Instruments and Methods in Physics Research, Section A: Accelerators, Spectrometers, Detectors and Associated Equipment 759 (2014) 16-22. doi 10.1016/j.nima.2014.04.062

[16] G. Dietze, H. Klein, Nuclear Instruments and Methods in Physics Research 193 (1982) 549-556. doi $10.1016 / 0029-554 X(82) 90249-X$. 
[17] J. M. Mueller, J. Mattingly, in: IEEE Nuclear Science Symposium Conference Record N2C1-3, 2015.

[18] R. Winyard, J. Lutkin, G. McBeth, Nuclear Instruments and Methods in Physics Research Section A 95 (1971) $141-153$.

[19] S. A. Pozzi, E. Padovani, M. Marseguerra, Nuclear Instruments and Methods in Physics Research, Section A: Accelerators, Spectrometers, Detectors and Associated Equipment 513 (2003) 550-558. doi 10.1016/j.nima. 2003.06 .012

[20] J. Allison, et al., IEEE Transactions on Nuclear Science 53 (2006) 270-278. doi $10.1109 /$ TNS. 2006.869826.

[21] E. Mendoza, D. Cano-Ott, T. Koi, C. Guerrero, IEEE Transactions on Nuclear Science 61 (2014) 2357-2364. doi:10.1109/TNS.2014.2335538.

[22] J. Randrup, R. Vogt, Physical Review C 80 (2009) 024601. doi 10.1103/ PhysRevC.80.024601 